Z. Klin. Chem. Klin. Biochem.

12. Jg. 1974, S. 98-102

\title{
Flammenphotometrische Bestimmung der freien Sulfationen im Serum
}

\author{
Von R. Leskovar und G. Weidmann
}

Aus der Balneologischen Forschungsstelle (̈̈rztlicher Leiter: Prof. Dr. R. Leskovar) des Staatsbades Bad Kissingen

(Eingegangen am 14. September/21. Dezember 1973)

\begin{abstract}
Es wird eine Methode beschrieben, in der im nichtenteiweißten Serum die freien Sulfationen durch eine im Überschuß zugegebene bekannte $\mathrm{BaCl}_{2}-\mathrm{Menge}$ ausgefällt werden. Die restlichen Barium-Ionen der Proben werden anschließend flammenphotometrișch bestimmt unter Verwendung eines natürlichen Serums als Standard, dessen Rest-Barium-Ionen-Konzentration vorher auf gravimetrischem Weg ermittelt wurde. Aus der Differenz von zugesetzter und der gemessenen Rest-Barium-Ionen-Konzentration berechnet sich der Sulfationen-Gehalt der Proben. Die optimalen Meßbedingungen wurden ermittelt und die Zuverlässigkeit der Methode überprüft. Die Durchführung der Untersuchungen kann sowohl manuell als auch halbautomatisch nach dem System Technicon „AutoAnalyzer" erfolgen. Folgender Mittelwert wurde bei 50 Kurpatienten festgestellt: 0,315 mmol/1 SO2- $\pm 0,031$ (s).
\end{abstract}

\section{Determination of free sulphate in serum by flame photometry}

A method is described in which the free sulphate ions of non-deproteinized serum are precipitated by the addition of an excess known quantity of $\mathrm{BaCl}_{2}$. The remaining barium ions of the sample are then determined by flame photometry, using a natural serum as a standard in which the residual barium ion concentration has been measured gravimetrically. The sulphate ion concentration of the sample is calculated from the difference between the added and the measured remaining barium ion concentrations. The optimal conditions for the method and its reliability are reported. The method may be performed manually, or semiautomatically using the Technicon „Autoanalyzer" System. An average of $0.315 \mathrm{mmol} / 1 \mathrm{SO}_{4}^{2-} \pm 0.031$ (s) was found for 50 patients under treatment.

In Verbindung mit den Studien über den Ionenhaushalt im menschlichen Körper, die auch im Rahmen der balneologischen Fragestellung von Bedeutung sind, wurde eine schnelle Analysenmethode zur Bestimmung der freien Sulfationen im Serum notwendig. Gravimetrische $(1,2)$, kolorimetrische $(3,4)$ und titrimetrische Verfahren (5) sind schwierig und zeitraubend. Neuerdings werden auch nephelometrische Verfahren $(6,7,8)$ verwendet, die aber wiederum eine recht umständliche Probenvorbereitung verlangen. Allen diesen Verfahren haftet außerdem der Nachteil an, daß sie große Substanzmengen für Routine-Untersuchungen vèrlangen, da sie wie die bisher entwickelten flammenphotometrischen (9, $10,11)$ und radiochemischen Verfahren (12) vom enteiweißten Serum ausgehen.

Im Folgenden wird eine flammenphotometrische Routinemethode zur Bestimmung der freien Sulfationen im nativen Serum beschrieben und über die Prüfung von Zuverlässigkeitskriterien berichtet. Die Methode kann bei gleichbleibender Vorbereitung der Proben und unter Verwendung derselben Standards sowohl manuell als auch halbautomatisch (AutoAnalyzer) durchgeführt werden.

\section{Material und Methoden \\ Manuelle Arbeitsweise \\ Geräte}

Flammenphotometer der Firma Eppendorf mit Barium-Filter $(510 \mathrm{~nm})$.
Einstellung des Verstärkungsreglers auf $\mathrm{V}=6$

Meßbereicheinstellung „100“

Brenngasgemisch: $500-520 \mathrm{~mm}$ Was̀sersäule Acetylen bei $0,5 \mathrm{kp} / \mathrm{cm}^{2}$ Luft

\section{Chemikalien}

Stammlösungen: $0,1 \mathrm{~mol} / 1 \mathrm{HCl}$

$$
0,05 \mathrm{~mol} / 1 \mathrm{BaCl}_{2} \text {-Lösung }
$$

\section{Reaktionslösung}

$2 \mathrm{mmol} / 1 \mathrm{BaCl}_{2}$ in $0,1 \mathrm{~mol} / 1 \mathrm{HCl}$ zur Fällung des Sulfates im Serum.

\section{Standardlösungen}

Blindlösung (Null-Lösung): Sammel-Serum mit 0,1 mol/1 HCl im Verhältnis 1:1 gemischt

Eichlösung $=$ Standard - Serum : Serum mit bekanntem RestBarium-Gehalt

\section{Herstellung des Standard-Serums}

Es wird eine größere Menge Serum mit der Reaktionslösung im Verhältnis 1:1 versetzt und einen Tag bei Raumtemperatur stehen gelassen. Der pH-Wert dieser Lösung beträgt etwa 3,5. Diese Acidität reicht aus, um das Mitfällen von Phosphaten zu verhindern. Das nach dieser Zeit ausgefallene Bariumsulfat wird abzentrifugiert, gewaschen und nach den üblichen Methoden der Gravimetrie weiter behandelt. Aus der Differenz von zugesetztem und gefälltem Barium errechnet sich die Rest-Barium-IonenKonzentration im Serum und daraus wiederum die Sulfationenmenge. Mit der Rest-Barium-Ionen-Konzentration dieser SerumLösung werden die Untersuchungsproben verglichen. Die von uns auf diesem Weg in 8 Sammelseren gefundenen Sulfationengehalte im nichtenteiweißten Serum zeigten einen Mittelwert $0,309 \mathrm{mmol} \pm 0,010(\mathrm{~s})$ und entsprechen den Werten anderer Autoren $(1,9,12,14)$, die nach vorheriger Enteiweißung des Serums festgestellt wurden. 


\section{Probenvorbereitung}

$1 \mathrm{ml}$ des Probenserums wird mit $1 \mathrm{ml}$ der Reaktionslösung in einem gut $4 \mathrm{ml}$ fassenden Plastikbecher (Technicon-AutoAnalyzer) versetzt und $24 \mathrm{~h}$ lang verschlossen mit einer Plastikkappe stehen gelassen. Darauf wird das ausgefallene Bariumsulfat bei $1000 \mathrm{~g} 5 \mathrm{~min}$ zentrifugiert. Damit ist die Probe zur Analyse fertig. Beim weiteren Arbeitsvorgang muß darauf geachtet werden, daß das im Becherboden befindliche $\mathrm{BaSO}_{4}$ nicht aufgerührt wird.

Der manuellen Ausführung der Analysen liegt die im Eppendorf Handbuch (15) beschriebene Arbeitsweise zugrunde. Mit der Null-Lösung wird der 0-Punkt elektrisch und mechanisch eingestellt. Mit der Eichlösung wird unter Benutzung des Feinreglers „Meßbereich" die Lichtmarke auf den Ba-Wert eingestellt, der dem Rest-Barium-Gehalt der Eichlösung entspricht. Als dazu gut geeignet erwies sich die Kalium-Skala (mval). Wird der Meßstrich 4 mval mit $2 \mathrm{mmol} /$ Ba gleichgestellt bzw. die Rest-Barium-Ionen-Konzentration des Eichserums auf den ihr zukommenden Wert, z. B. 1,7 $\mathrm{mmol}$ auf den 3,4 mval Skalenstrich eingestellt, so entspricht jeder Teilstrich der Skala 0,10 mval d. h. 0,05 mmol Barium. Liest man in umgekehrter Richtung, also von 4,0-0, so ergibt die jeweilige Differenz den Sulfatgehalt in mval. Es ist zweckmäßig, die Einstellung des 0-Punktes sowie des Eichpunktes nach 5 Probenmessungen zu wiederholen (vgl. auch Tabelle 2).

Automatische Ausführung der Analysen

Geräte

Flammenphotometer (wie oben)

Probennehmer II der Firma Technicon, AutoAnalyzer : Nockenscheibe 50 Proben/h, Verhältnis Probe-Spülung 1:2,5.

Proportionierschlauchpumpe. Wir benutzten die Schlauchpumpe der Firma Desaga, bei der die Kanäle für die Pumpenschläuche (Fa. Technicon) in der ganzen Länge freigemacht werden müssen. Pumpengeschwindigkeit: „90“.

Zur Entfernung der Luftblasen wird knapp vor dem Saugstutzen des Flammenphotometers ein C 5 Glas-Fitting der Fa. Technicon als „Debubbler" angebracht.

Materialverbrauch je Probe: $1,6 \mathrm{ml} \pm 0,2 \mathrm{ml}$, davon die Hälfte Serum. Fließdiagramm siehe Abbildung 1.

Wie aus dem Fließdiagramm hervorgeht, wird die Probe unverändert photometriert. Als Spülflüssigkeit dient $0,1 \mathrm{~mol} / 1 \mathrm{HCl}$. Um bessere Meßwerte zu erzielen, empfiehlt es sich, das Flammenphotometer vorher eine Stunde lang laufen zu lassen. Zur Herstellung eines Gleichgewichts in der Zerstäuberglocke, das sich in einer Beruhigung des Flammenuntergrundes (0-Linie) bzw. der gleichmäßigeren Ausschlaggrößen kundtut und durch einen gallertartigen Belag an der Wandung der Zerstäuberglocke sichtbar wird, sollen zunächst 5 Standard (Eich)-Seren den eigentlichen Messungen vorausgeschickt werden.

Zur rechnerischen Auswertung muß der Flammenuntergrund, d. h. der für das Leerserum erhaltene Ausschlag von den für die Proben und das Standard-Serum erhaltenen Ausschlagweiten abgezogen werden. Dann bildet man aus den Ausschlagweiten (I) von Probe und Standard den Quotienten $=$ F. Wie sich

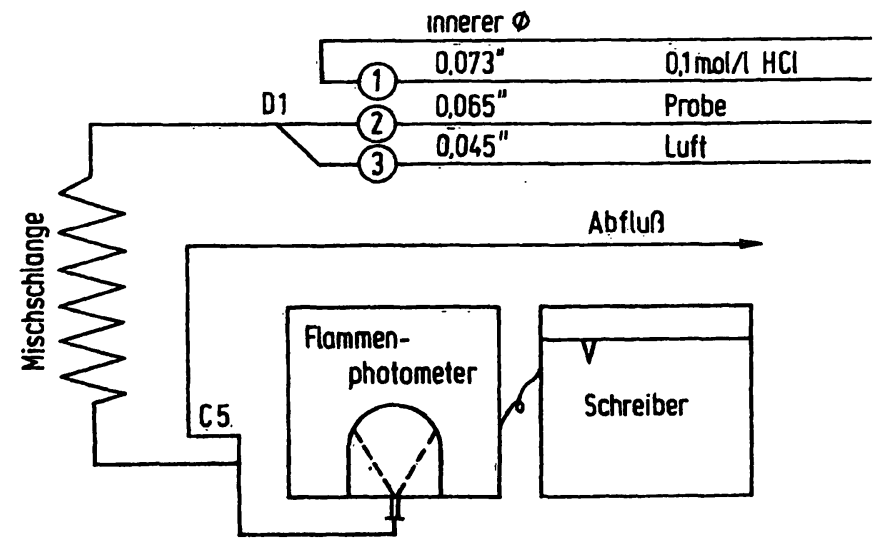

Abb. 1. Fließdiagramm Sulfat im Serum. leicht ableiten läßt, ergibt sich die Berechnung des Sulfatgehaltes nach:

$$
\begin{aligned}
& a-(F \cdot c)=G \\
& a=\text { Zusatz an Ba mmol/1 zur Probe }(=2 \mathrm{mmol} / \mathrm{Ba} / \mathrm{l}) \\
& F=\frac{I(\text { Probe })}{I(\text { Standard })} \\
& c=\begin{array}{l}
\text { Rest-Barium-Konzentration im Standard-Serum in } \\
\text { mmol } / 1
\end{array} \\
& G=\text { Sulfatgehalt in der Probe in mmol } / \mathrm{l} .
\end{aligned}
$$

Aufgrund des geschilderten Vorgehens stellten wir bei $50 \mathrm{Kur}-$ patienten - vorwiegend Magenkranke - einen Serum-Sulfatgehalt von $\overline{\mathrm{X}}=0,315 \mathrm{mmol} / \mathrm{l} \pm 0,0315$ (s) fest.

\section{Ergebnisse und Diskussion}

\section{Prüfung der Methode}

Die Zuverlässigkeit sowohl der manuellen als auch der automatischen Arbeitsweise ergibt sich einerseits aus der auf flammenphotometrischem Weg bei gegebener Apparatur überhaupt erreichbaren Nachweisgrenze, andererseits aus der Reproduzierbarkeit der Werte. Die Nachweisgrenze wird von der Herstellerfirma mit $2 \mathrm{mg} / 1$ $\mathrm{Ba}$, also $0,0145 \mathrm{mmol} / 1$, angegeben. In diesen Bereich reichen kaum einige Standardabweichungen unserer Serum-Sulfatmessungen.

\section{Barium-Emissionskurven}

Zunächst muß festgestellt werden, wie sich

a) die Emissionskurve von reinen Ba-Lösungen in den anzuwendenden Konzentrationen verhält,

b) ob die Bariumenmission von den im Serum vorhandenen Ionen und

c) ob sie vom Serum selbst beeinflußt wird.

$\mathrm{zu}$ a) Die Messung von Ba-Lösungen in $0.1 \mathrm{~mol} / 1 \mathrm{HCl}$ mit steigendem Ba-Gehalt ergab eine geradlinig verlaufende Eichkurve, die durch den 0-Punkt des Koordinatensystems geht (s. Kurve a in Abb. 2).

zu b) Werden diese Ba-Lösungen auf ein Elektrolytgemisch, das dem des menschlichen Serums entspricht, aufgestockt - wir benutzten dazu die Original-Standardlösung der Fa. Eppendorf - so hebt sich zunächst der Flammenuntergrund. Zieht man den Wert, der auf die Elektrolyte entfällt ab, so ergibt das aufgestockte Barium wiederum eine gerade, durch den 0-Punkt verlaufende Linie, die jedoch etwas steiler als die reine Ba-Lösungslinie verläuft (s. Kurve b in Abb. 2).

zu c) Für die Untersuchungen des Verhaltens des Bariums im Serum wurden aliquote Teile eines Sammelserums, dessen $\mathrm{SO}_{4}$-Gehalt vorher auf gravimetrischem Weg ermittelt wurde, mit abgestuften $\mathrm{BaCl}_{2}$-Mengen $(0,5$ bis $2,5 \mathrm{mmol} / \mathrm{l})$ versetzt und in der beschriebenen Weise flammenphotometrisch bestimmt. Bei unserem Sammelserum-Sulfatgehalt von $0,31 \mathrm{mmol} / 1 \mathrm{SO}_{4}$ waren somit die entsprechenden zu erwartenden Restbariumwerte bei $\mathrm{Zu}$ gabe von $0,5-, 1,0-, 1,5-, 2,0-, 2,5 \mathrm{mmol} \mathrm{Ba} / 1 \mathrm{je}$ weils um $0,31 \mathrm{mmol} \mathrm{Ba/1}$ niedriger, also 0,19 . 0,69-, 1,19-, 1,69-, 2,19 mmol Ba/l. Die photo- 


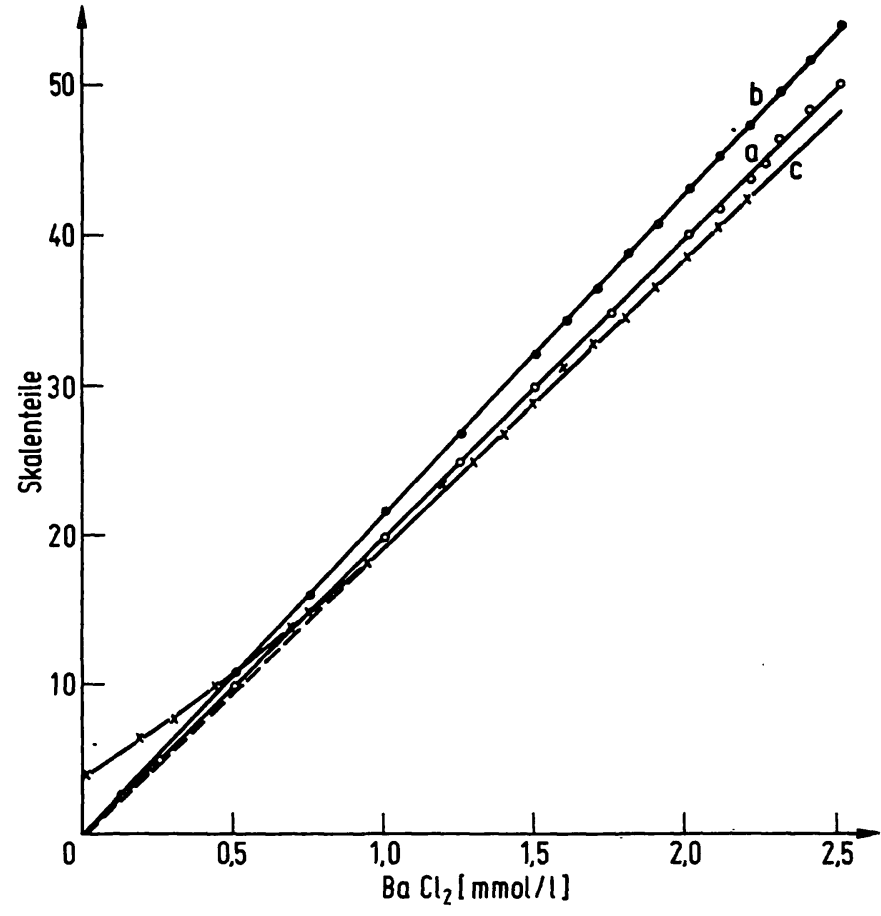

Abb. 2. Barium-Eichkurven.

a) $\bigcirc-0=$ reine $\mathrm{BaCl}_{2}$-Lösungen.

b) $\bullet-\bullet=$ auf ein Elektrolytgemisch, das dem des menschlichen Serums entspricht, aufgestockte $\mathrm{BaCl}_{2}$-Lösungen.

c) $x-x-x=$ dem Serum zugegebene $\mathrm{BaCl}_{2}$-Lösungen (Näheres vgl. Text).

metrisch angezeigten Meßwerte werden nun an diesen Punkten der Abszisse angesetzt. So entstand die Kurve $c$ in Abbildung 2.

Dieselbe Bariumkonzentration weist somit beim Serum um rund $10 \%$ geringere Emissionsausschläge auf als bei einer entsprechend zusammengesetzten wäßrigen Elektrolytlösung (,,Eppendorf-Standard“).

Außerdem verläuft die Kurve nur im höheren Konzentrationsbereich, also zwischen 1 und $2,5 \mathrm{mmol} \mathrm{Ba} / \mathrm{l}$, geradlinig auf den 0-Punkt zu. Im unteren Teil biegt sich sich nach oben durch, d.h. die Ausschläge sind zu groß.

Zur Erklärung dieser Erscheinung kann nur angenommen werden, daß kleinere Ba-Mèngen als $1 \mathrm{mmol}$ $\mathrm{Ba} / \mathrm{l}$ das Sulfat im Serum nicht mehr quantitativ ausfällen.

Daraus ergibt sich die Folgerung, Messungen im Serum so anzusetzen, daß eine Rest-Barium-Ionen-Konzentration von $1,0 \mathrm{mmol} / \mathrm{l}$ nicht unterschritten wird.

\section{Reproduzierbarkeit der Methode}

Im selben Serum wurde Sulfat teils hintereinander, teils in Abständen von einiǵen Tagen nach der automatisierten Methode bestimmt. Nachdem die Meßwerte auf ein Standard-Serum bezogen wurden, wurde mit Hilfe von Variations-Koeffizienten untersucht, ob und inwieweit sich eine häufigere Dazwischenschaltung von Standardseren auf die Genauigkeit der Meßergeb- • nisse auswirkt. Die Ergebnisse sind aus Tabelle 1 ersichtlich. Aus der nächsten Tabelle (Tab. 2) geht die
Tab. 1. Sulfatbestimmungen im selben Serum mit 0,308 $\mathrm{mmol} / \mathrm{l}$ $\mathrm{SO}_{4}^{2-}$ (gravimetrisch) bei verschieden-häufiger StandardZwischenschaltung.

\begin{tabular}{lllll}
\hline $\begin{array}{l}\text { Standard } \\
\text { auf Anzahl } \\
\text { der Proben }\end{array}$ & $\mathrm{n}$ & \multicolumn{2}{c}{$\mathrm{SO}_{4}^{2-}[\mathrm{mmol} / \mathrm{l}]$} \\
$\pm \mathrm{s}$ & $\begin{array}{l}\text { VK } \\
{[\%]}\end{array}$ \\
\hline $1: 20$ & 62 & 0,335 & 0,0218 & 6,72 \\
$1: 10$ & 60 & 0,3025 & 0,0133 & 4,40 \\
$1: 5$ & 57 & 0,3052 & 0,0148 & 4,84 \\
$1: 3$ & 58 & 0,3125 & 0,0142 & 4,55 \\
$1: 1$ & 59 & 0,3171 & 0,0194 & 5,35 \\
\hline
\end{tabular}

Tab. 2. Reproduzierbarkeit der Meßwerte (Tag zu Tag-Bestimmungen) nach der manuellen (1) und der automatischen (2) Arbeitsweise

\begin{tabular}{|c|c|c|c|c|c|c|}
\hline \multirow{2}{*}{ Methode } & \multirow{2}{*}{$\mathbf{n}$} & \multicolumn{2}{|c|}{$-\mathrm{SO}_{4}^{2-}[\mathrm{mmol} / \mathrm{l}]$} & \multirow{2}{*}{$\begin{array}{l}\text { VK } \\
\text { [\%] }\end{array}$} & \multirow{2}{*}{$\begin{array}{l}\mathrm{SO}_{4}^{2-} \\
\text { [mmol/1] } \\
\text { gravime- } \\
\text { trisch }\end{array}$} & \multirow{2}{*}{$\begin{array}{l}\text { Differenz } \\
\text { Meßwert- } \\
\text { Sollwert } \\
\text { in \% }\end{array}$} \\
\hline & & $\overline{\mathbf{x}}$ & $\pm \mathrm{s}$ & & & \\
\hline 2 & $\begin{array}{l}13 \\
57\end{array}$ & $\begin{array}{l}0,292 \\
0,305\end{array}$ & $\begin{array}{l}0,0183 \\
0,0148\end{array}$ & $\begin{array}{l}6,3 \\
4,84\end{array}$ & $\begin{array}{l}0,298 \\
0,308\end{array}$ & $\begin{array}{l}2,0 \\
1,0\end{array}$ \\
\hline
\end{tabular}

Reproduzierbarkeit der Meßwerte nach der manuellen (1) und der automatischen (2) Arbeitsweise in Tag zu Tag-Bestimmungen hervor.

Erwartungsgemäß ist bei Anwendung der automatischen Arbeitsweise der Variationskoeffizient etwas niedriger als bei der manuellen. Die Abweichungen der flammenphotometrisch von den gravimetrisch bestimmten Werten betragen nur 2 bzw. $1 \%$.

\section{Wiederfindungsversuche}

Diese wurden durchgeführt durch

a) Zugabe von abgestuften Ba-Mengen,

b) Zugabe von abgestuften $\mathrm{SO}_{4}$-Mengen zum Serum.

$\mathrm{zu}$ a) Einem Serum, dessen $\mathrm{SO}_{4}$-Gehalt durch gravimetrische Bestimmung bekannt war $(0,308 \mathrm{mmol} / 1)$, wurden ansteigende Mengen $\mathrm{BaCl}_{2}$ zugegeben und dann dessen $\mathrm{SO}_{4}$-Gehalt photometrisch bestimmt. Das Ergebnis ist aus Tabelle 3 ersichtlich. Bei Konzentrationen unter $0,75 \mathrm{mmol} / 1 \mathrm{Ba}(=\mathrm{Zu}$ gabe von $1 \mathrm{mmol} / 1 \mathrm{Ba})$ tritt die schon besprochene Abweichung (vgl. Abb. 2) der Meßwerte in Erscheinung.

zu b) Demselben, mit $2 \mathrm{mmol} / \mathrm{l}$ Ba versetzten Serum werden abgestufte Mengen an Schwefelsäure zugegeben. Wie aus der Tabelle 4 hervorgeht, entsprechen die Wiederfindungsraten den $\mathrm{SO}_{4}-\mathrm{Zu}$ gaben, jedoch nur bis zu einer Rest-Barium-IonenKonzentration von etwa $0,75 \mathrm{mmol} / 1 \mathrm{Ba}$. Man kommt - unter Berücksichtigung des serumeigenen Sulfat-Gehaltes - somit auch hier wieder zu der Feststellung, daß bei geringer Ba-Konzentration die Reaktion nicht mehr vollständig abläuft.

Z. Klin. Chem. Klin. Biochem. / i 12 Jahrg. 1974 / Heft 3 
Tab. 3. Bestimmung des Sulfat-Gehaltes desselben Serums (mit $0,308 \mathrm{mmol} / 1 \mathrm{SO}_{4}$ ) bei abgestufter $\mathrm{BaCl}_{2}-\mathrm{Zugabe}$.

\begin{tabular}{|c|c|c|c|c|c|}
\hline $\begin{array}{l}\mathrm{BaCl}_{2}^{-} \\
\text {Zugabe } \\
\mathrm{mmol} / \mathrm{l}\end{array}$ & $\mathbf{n}$ & $\bar{x}^{\mathrm{SO}_{4}^{2-}}$ & $\begin{array}{l}\text { ol/1] } \\
\pm s\end{array}$ & $\begin{array}{l}\text { VK } \\
\text { [\%] }\end{array}$ & $\begin{array}{l}\text { Differenz } \\
\text { Meßwert- } \\
\text { Sollwert } \\
\text { in \% }\end{array}$ \\
\hline $\begin{array}{l}2,5 \\
2,4 \\
2,3 \\
2,2 \\
2,1\end{array}$ & $\begin{array}{l}12 \\
10 \\
13 \\
10 \\
10\end{array}$ & $\begin{array}{l}0,3179 \\
0,3460 \\
0,3123 \\
0,3165 \\
0,3240\end{array}$ & $\begin{array}{l}0,0278 \\
0,0346 \\
0,0211 \\
0,0190 \\
0,0131\end{array}$ & $\begin{array}{l}8,75 \\
8,75 \\
6,77 \\
6,00 \\
4,02\end{array}$ & $\begin{array}{r}+3,2 \\
+\quad 2,2 \\
+1,3 \\
+3,0 \\
+\quad 5,0\end{array}$ \\
\hline 2,0 & - & - & - & - & - \\
\hline 1,9 & 14 & 0,2985 & 0,0217 & 7,28 & $-2,5$ \\
\hline 1,8 & 14 & 0,3070 & 0,0183 & 5,98 & 0 \\
\hline 1,7 & 11 & 0,3135 & 0,0189 & 6,06 & $+1,5$ \\
\hline 1,6 & 12 & 0,3120 & 0,0201 & 6,45 & $+1,3$ \\
\hline 1,5 & 12 & 0,2925 & 0,0241 & 8,24 & $-4,8$ \\
\hline 1,25 & 14 & 0,3060 & 0,0233 & 7,61 & 0 \\
\hline 1,0 & 16 & 0,2730 & 0,0271 & 9,90 & $-11,3$ \\
\hline 0,75 & 15 & 0,2440 & 0,0383 & 15,60 & $-20,5$ \\
\hline 0,5 & 14 & 0,1970 & 0,1047 & 53,00 & $-36,0$ \\
\hline
\end{tabular}

Tab. 4. Zugabe abgestufter Sulfatmengen zu demselben mit $2 \mathrm{mmol} / 1 \mathrm{Ba}$ versetzten Serum (mit $0,30 \mathrm{mmol} / 1$ eigenem Sulfatgehalt) Mittelwerte von je 7 Messungen.

\begin{tabular}{|c|c|c|c|c|c|c|}
\hline \multirow{2}{*}{$\begin{array}{l}\text { Zugabe } \\
\text { von } \\
\mathrm{SO}_{4}^{2-} \\
{\left[\mathrm{mmol}^{2}\right]}\end{array}$} & \multicolumn{2}{|c|}{$\begin{array}{l}\text { Ba-Restgehalt } \\
\text { [mmol/1] }\end{array}$} & \multicolumn{2}{|c|}{$\begin{array}{l}\text { entspricht } \\
\mathrm{SO}_{4}^{2-}[\mathrm{mmol} / 1]\end{array}$} & \multirow[t]{2}{*}{$\begin{array}{l}\text { VK } \\
\text { [\%] }\end{array}$} & \multirow{2}{*}{$\begin{array}{l}\text { Differenz } \\
\text { Sollwert- } \\
\text { Meßwert } \\
\text { in \% }\end{array}$} \\
\hline & rechn. & funden & $\overline{\mathbf{x}}$ & $\pm \mathrm{s}$ & & \\
\hline 0 & - & 1,70 & 0,30 & - & - & - \\
\hline 0,25 & 1,45 & 1,434 & 0,266 & 0,0188 & 1,30 & $+6,4$ \\
\hline 0,50 & 1,20 & 1,183 & 0,518 & 0,01 & 1,94 & $+3,5$ \\
\hline 0,75 & 0,95 & 0,920 & 0,780 & 0,0087 & 0,94 & $+4,0$ \\
\hline 1,00 & 0,70 & 0,671 & 1,029 & 0,0229 & 3,40 & $+3,0$ \\
\hline 1,25 & 0,45 & 0,4092 & 1,291 & 0,0127 & 3,10 & $+3,2$ \\
\hline 1,50 & 0,20 & 0,209 & 1,492 & 0,0154 & 7,40 & $-0,6$ \\
\hline 1,75 & 0 & 0,087 & 1,613 & 0,0075 & 8,60 & $-0,7$ \\
\hline 2,00 & 0 & 0,050 & 1,650 & 0,0116 & 23,20 & $-1,7$ \\
\hline
\end{tabular}

\section{Weitere Beobachtungen}

Nachdem unsere Methode auf einer Ausfallung von $\mathrm{BaSO}_{4}$ beruht, mußte zunächst der Zeitpunkt bestimmt werden, an dem die Reaktion zwischen den Ba- und den Sulfationen abgelaufen ist.

Es wurde eine größere Menge Serum mit $2 \mathrm{mmol} / \mathrm{l} \mathrm{Ba}$ bei Raumtemperatur versetzt. In bestimmten Zeitabständen wurde sodann die Rest-Barium-Ionen-Konzentration bestimmt (s. Tab. 5). Das Ende des Reaktionsablaufes kann somit nach 16 Stunden angenommen werden; der Mittelwert der Bestimmungen schwankt ab diesem Zeitpunkt im selben Ausmaß um den gravimetrisch festgestellten Wert von $0,323 \mathrm{mmol} / 1 \mathrm{SO}_{4}^{2-}$ wie bei vorhergehenden Meßreihen.

Als Grundlage der Messungen dient ein Standard-Serum bekannter $\mathrm{SO}_{4}$-Konzentration, mit dem die Proben flammenphotometrisch verglichen werden. Infolgedessen sind auch die Meßergebnisse in erster Linie von der Genauigkeit der auf gravimetrischem Weg bestimmten Rest-
Tab. 5. Bestimmung des Reaktions-Endes zwischen zugesetzten Ba-lonen und freien Sulfationen im selben Serum (mit gravimetrisch bestimmtem Wert $0,322 \mathrm{mmol} / 1 \mathrm{SO}_{4}^{27}$ ).

\begin{tabular}{llllr}
\hline $\begin{array}{l}\text { Stunden } \\
\text { nach } \\
\text { Ba-Zusatz }\end{array}$ & $\mathrm{n}$ & $\overline{\mathrm{x}}$ & $\mathrm{SO}_{4}^{2-}[\mathrm{mmol} / \mathrm{l}]$ \\
$\pm \mathrm{s}$ & $\begin{array}{r}\mathrm{VK} \\
{[\%]}\end{array}$ \\
\hline 1 & 7 & 0,1868 & 0,0421 & 22,6 \\
2 & 4 & 0,2463 & 0,0189 & 9,2 \\
3 & 4 & 0,2179 & 0,0139 & 8,7 \\
4 & 3 & 0,2603 & 0,0201 & 7,6 \\
6 & 3 & 0,2498 & 0,0134 & 5,3 \\
8 & 5 & 0,2642 & 0,0405 & 15,3 \\
10 & 3 & 0,3035 & 0,0054 & 1,8 \\
16 & 3 & 0,3165 & 0,0071 & 2,2 \\
18 & 3 & 0,3213 & 0,0112 & 3,5 \\
20 & 4 & 0,3378 & 0,0201 & 5,9 \\
24 & 3 & 0,3150 & 0,0152 & 4,7 \\
nach & & & & \\
2 Tagen & & 0,3285 & 0,0187 & 5,5 \\
und mehr & & & & \\
\hline
\end{tabular}

Barium-Ionen-Konzentration des Standard-Serums abhängig. Die Richtigkeit des Serumstandards kann aufgrund der Abbildung 2 kontrolliert werden. Die Ausschläge der Kurve $c$ (Serum) sind um etwa $10 \%$ geringer als die der Kurve $b$ (Eppendorf-Standard mit aufgestocktem $\mathrm{Ba}$ ). Mit einem entsprechenden reinen Elektrolytstandard, z. B. Eppendorf-Standard mit $1,5 \mathrm{mmol} / 1 \mathrm{Ba}$ kann sofort Kurve $b$ und daraus Kurve $\mathrm{c}$ konstruiert werden, aus der dann der Serum-Sulfatgehalt abgelesen werden kann.

Vergleicht man den Mittelwert unserer 8 Sammelseren von $0,309 \mathrm{mmol} / 1 \pm 0,010$ (s) Sulfat mit den Werten anderer Autoren, so stimmen sie gut überein. So fanden Berglund und Sörbo (8) einen Mittelwert von $0,33 \mu \mathrm{mol} / \mathrm{ml}$ mit einem Bereich von 0,21-0,48, Guerrero (16) 0,47 $\pm 0,15$ mval, Hinsberg-Lang (1) geben $0,7-1,7 \mathrm{mg} \% \mathrm{~S}$, was $0,22-0,53 \mathrm{mmol} / 1$ Sulfat entspricht, an, Miller et al (12), deren Ergebnisse auch von Dokumenta Geigy (14) angegeben werden, fanden einen Mittelwert von 0,645 $\pm 0,17 \mathrm{mval} / 1$.

Vergleicht man jedoch unseren Sammelserum-Wert mit den an 50 Kurpatienten - vorwiegend Magenkranken gewonnenen Werten $0,315 \pm 0,0315 \mathrm{mmol} / 1$, so fällt die etwas geringere Sulfatkonzentration der Sammelseren auf. Nun stammen die Sammelseren, die uns freundlicherweise von der Leberklinik Prof. Kalk abgetreten wurden, von Leberpatienten. Es dürfte daher der bei magenkranken Kurpatienten gefundene Mittelwert dem Normalwert, der unserer Methode entspricht, näherliegon.

Bei richtiger Aufbewahrung (Kühlschrank) blieb der Sulfatgehalt unseres Standard-Serums im Verlauf von drei Monaten unverändert.

Wie aus Tabelle 3 hervorgeht, ergibt die automatisierte Arbeitsmethode (VK 5\%) etwas bessere Ergebnisse als die manuelle (VK 6\%), beide liegen jedoch wesentlich unter der für klinische Analysen noch zugelassenen Grenze von $10 \%(16)$. 


\section{Literatur}

1. Hinsberg, K. \& Lang, K. (1957), Medizinische Chemie für den klinischen und theoretischen Gebrauch. Verlag UrbanSchwarzenberg/München.

2. Waelsch, H. \& Klepetar, G. (1932), Hoppe Seylers Z. Physiol. Chem. 211, 47-56.

3. Bertolacini, R. J. \& Barney, J. E. (1958), Anal. Chem. 30, 202-205.

4. Häkkinen, J. P. \& Häkkinen, L. M. (1959), Scand. J. Clin. Invest. 11, 294-297.

5. Baudisch, J., Beilstein, G. \& Neuenhausen, H. E. (1968), Fresenius Z. Analyt Chem. 235, 231-234.

6. Dogson, K. S. (1961), Biochem. J. 78, 312-319.

7. Ferrera, L. W., Floyd, R. S. \& Blanchar, R. W. (1965), Sonderdruck R 623 Technicon (65-PJ 27) New York.

8. Berglund, F. \& Sörbo, B. (1960), Scand. J. Clin. Lab. Invest. 12, 147-153.

9. Strickland, R. D. \& Maloney, Clara M. (1954), Am. J. Clin. Pathol. 24, 1100-1102.
10. Burriel-Marti, F., Ramirez-Munos, J. \& Rexach, M. L. (1957), Anal. Chem. Acta 17, 559-569.

11. Herbolsheimer, R. (1963), Freșenius Z. Analyt. Chem. 201, 418.

12. Miller, E., J. Hlad, L., Stanley.Levine, M. S., Holmers, J. H. \& Elrick, H. (1961), Labor. Methods 58, 656-661.

13. Herrmann, R. \& Alkemade, Th. J.: Flammenphotometrie (1960), Springer Verlag, Berlin.

14. Geigy, J. R. (1968), Documenta Geigy. Wissenschaftliche Tabellen.

15. Eppendorf-Gerätebau Netheler und Hinz GmbH (1965), Handbuch Flammenphotometer-Eppendorf.

16. Guerrero, S. B., zit. Bersin Th. (1963), Biochemie der Mineral- und Spurenelemente, Akad. Verlagsgesellschaft, Frankfurt/Main.

17. Richterich, R. (1968), Klinische Chemie, Akad. Verlagsges. Frankfurt/M.

Prof. Dr. R. Leskovar 8730 Bad Kissingen

Balneologische Forschungsstelle Prinzregentenstraße 2 